\title{
How humans evolved large brains: Comparative evidence
}

\author{
Isler, Karin ; van Schaik, Carel P
}

\begin{abstract}
The human brain is about three times as large as that of our closest living relatives, the great apes. Overall brain size is a good predictor of cognitive performance in a variety of tests in primates.[1, 2] Therefore, hypotheses explaining the evolution of this remarkable difference have attracted much interest. In this review, we give an overview of the current evidence from comparative studies testing these hypotheses. If cognitive benefits are diverse and ubiquitous, it is possible that most of the variation in relative brain size among extant primates is explained by variation in the ability to avoid the fitness costs of increased brain size (allocation trade-offs and increased minimum energy needs). This is indeed what we find, suggesting that an energetic perspective helps to complement approaches to explain variation in brain size that postulate cognitive benefits. The expensive brain framework also provides a coherent scenario for how these factors may have shaped early hominin brain expansion.
\end{abstract}

DOI: https://doi.org/10.1002/evan.21403

Posted at the Zurich Open Repository and Archive, University of Zurich

ZORA URL: https://doi.org/10.5167/uzh-97922

Journal Article

Accepted Version

Originally published at:

Isler, Karin; van Schaik, Carel P (2014). How humans evolved large brains: Comparative evidence. Evolutionary Anthropology, 23(2):65-75.

DOI: https://doi.org/10.1002/evan.21403 


\section{How humans evolved large brains: comparative evidence}

\section{Karin Isler and Carel P. van Schaik}

Anthropological Institute and Museum, University of Zürich-Irchel Winterthurerstrasse 190, CH-8057 Zürich, Switzerland

Email: kisler@aim.uzh.ch

Phone: 0041446355401

Fax: 0041446356804

Key words: energetics, comparative phylogenetic analysis, hominin evolution

\section{About the authors}

Karin Isler studies brain size evolution in mammals and birds using a phylogenetic comparative approach. She has published a series of papers on energetic correlates of brain size evolution, and is currently investigating cognitive and physiological strategies to buffer lean periods.

Carel van Schaik is interested in social, cognitive and cultural evolution of primates, focusing specifically on explaining traits that are unusually developed in humans. $\mathrm{He}$ currently focuses on comparative studies of cooperative breeding and cooperative hunting to identify convergent developments in the hominin lineage. 
The human brain is about three times as large as that of our closest living relatives, the great apes, and overall brain size is a good predictor of cognitive performance in a variety of tests in primates. ${ }^{1,2}$ Therefore, hypotheses explaining the evolution of this remarkable difference have attracted much interest. In this review, we aim to give an overview of the current evidence from comparative studies testing these hypotheses. If cognitive benefits are diverse and ubiquitous, it is possible that most of the variation in relative brain size among extant primates is explained by variation in the ability to avoid the fitness costs of increased brain size (allocation tradeoffs and increased minimum energy needs). This is indeed what we find, suggesting that an energetic perspective helps to complement approaches to explain variation in brain size that postulate cognitive benefits. The expensive brain framework also provides a coherent scenario for how these factors may have shaped early hominin brain expansion.

During most of the past 30 years, there were two major approaches to explain the evolution of larger brains relative to the overall trend with body size. Although analyses of evolutionary changes in the size of a structure rarely need to be concerned about the energetic costs of its production or maintenance, one approach has stressed that due the high metabolic costs of brain tissue, ${ }^{3}$ brains can only increase in size if the additional energy costs can somehow be met. ${ }^{4}$ Another approach, while recognizing these costs, focused on the enhanced cognitive abilities in the ecological or social domain of larger brains, and on the fitness benefits this brings to their owners, by increasing survival or reproductive output. ${ }^{5}$ Because selection can only favor an increase in the size of a structure relative to its ancestral state if the net balance of fitness costs and benefits is positive (Figure 1), it is clear that the two approaches necessarily complement each other.

The first aim of this review is to integrate costs and benefits approaches in a predictive model to explain the existing variation in brain size by taking a broad comparative perspective, considering primates and other mammals, and birds where necessary (see also ${ }^{6}$ ). Each species does not just occupy an ecological niche, it also constructs it by influencing the 
external conditions. ${ }^{7}$ An increase in brain size may change the conditions, and when such evolutionary feedback loops occur, cause and effect become impossible to disentangle. As we look at the current endpoints of a long evolutionary history, such a model cannot and should not look for causal pathways, but only for patterns of correlated evolution. As a result, although the cost and the benefit approaches obviously complement each other, their combination in a predictive model is not trivial since many correlates of brain size can be interpreted either way. For example, high quality diet may indicate increased digestive efficiency and thus be an independent external determinant that is relevant for understanding brain size variation from an energetic perspective, but on the other hand it may also reflect a cognitive benefit of an enhanced ability to find or gain access to high-quality food items. Therefore, most studies so far have concentrated on one or the other approach, and until recently most focused on benefits. ${ }^{8}$ Here, we place the emphasis on reviewing the empirical evidence for energetic correlates of brain size evolution. We discuss each potential correlate separately and then test whether a combination of various factors explains more of the existing variation in primate brain size than any single factor.

Our second aim is to apply these insights to human evolution. Starting from a statistical model for nonhuman primates that includes both benefits and costs, we include humans to see whether the characteristics of our species fit the general primate pattern. At the end, by looking at fossil and archeological evidence for the order of appearance of these human-specific traits, we present the most parsimonious historical scenario given current comparative evidence.

\section{Benefit approaches}

Several behavioral characteristics have been suggested as the specific selective agent that enabled a decrease in mortality, and ultimately an extension of the maximum observed lifespan, in relatively large-brained species. The hypotheses can broadly be divided into focusing on either social or ecological benefits of enhanced cognitive abilities (reviewed for 
example in ${ }^{9}$ ). Social skills comprise social strategizing or pair-bonding, ${ }^{5,10,11}$ whereas ecological skills are spatial orientation to find food patches or remember their location, dietary flexibility, food processing, tool use or extractive foraging, ${ }^{12,13}$ or predator recognition and avoidance. ${ }^{14}$

The best known benefits-oriented explanation of brain size evolution argues that the social environment is likely to provide the context in which behavioral flexibility is most beneficial. ${ }^{15}$ Thus, the Social Brain hypothesis proposed that living in large groups is only feasible if the animals are able to monitor and remember social relationships. This idea thus links increased brain size to the evolution of large and complex social groups in primates. ${ }^{15}$ In non-primates, this group-size prediction was supported for some lineages but not others. ${ }^{10}$ Therefore, the hypothesis was slightly modified to argue that in birds or mammals other than primates, the most complex form of a social relationship is the pair bond, which explains the positive correlation between the occurrence of pair-bonds and brain size in artiodactyls and carnivores ${ }^{10}$ as well as birds. ${ }^{11}$ Because primates have friendships that function like the pair bonds in other lineages, the primate pattern also fits this new explanation. The social brain hypothesis also explains why over evolutionary time, those lineages that had social bonds or pair bonds showed steep increases over time, whereas those that did not, failed to do so. ${ }^{8}$

Although the Social Brain hypothesis explains much of the variation of relative brain (and especially neocortex) size in cercopithecoid primates, it does not account for some striking grade shifts. ${ }^{16}$ For instance, lemurs appear to experience similar social challenges but are clearly less encephalized than monkeys. ${ }^{17}$ Therefore, we will pursue the idea that some grade shifts arise because of problems generating sufficient energy or a large enough survival benefit for selection to have favored increased brain size.

\section{Ecology and general flexibility}

A relatively large brain may evolve preferentially in those species or lineages that rely on a variety of cognitive skills to obtain food or avoid predators or parasites. The causal 
link is straightforward: more skilled individuals acquire more or better food and are better at avoiding starvation, predation or infections, thus surviving longer and producing more surviving offspring. ${ }^{18}$ This idea is supported by correlations between survival rates, invasion success, or longevity on the one hand and brain size or innovation rates on the other hand. ${ }^{19,20}$ However, if survival is increased, life history theory predicts that other aspects of life history will follow suit. ${ }^{21}$ The evidence of prolonged development periods in larger-brained species $^{9,22}$ may thus simply be an inevitable consequence of prolonged lifespan. Attempts to determine which of the life-history variables is most closely linked to brain size, and would be the most likely driver of the correlations between brain and life history in evolution, are therefore rather futile. All life-history traits covary, and therefore the results of multivariate regression analyses are mostly determined by the different amounts of error variation within each variable. The variable measured with the highest accuracy will turn out to explain most of the variation in brain size, while others are dropped from the model. Consequently, studies of different datasets or using slightly different methodology may yield divergent results. ${ }^{23}$

Specialized skills such as food caching, tool use or manual dexterity have so far not been related to overall brain size, but to cerebellum size, the degree of cortical folding ${ }^{24,25}$ or the relative size of different brain parts. ${ }^{26}$ Nonetheless, there is good evidence that high-level cognitive flexibility, applied to solve problems in any domain, is strongly correlated with overall brain size, at least across primate species. ${ }^{1,2}$ Independent of the specific ecological context, enhanced brainpower therefore is expected to entail a survival benefit. ${ }^{27}$ This makes most sense if a social component is added ${ }^{28}$ which also makes it highly compatible with the social brain hypothesis: especially if innovative solutions are made heritable through vertical or oblique social transmission would we expect individual intelligence to produce a selective advantage. This could explain why some semi-solitary species that exhibit prolonged motheroffspring contact but do not need social skills like opportunistic coalitions (such as orangutans) are nevertheless large-brained, whereas some species living in complex groups with low social tolerance (such as hyenas) apparently did not evolve larger brains. ${ }^{16}$ 


\section{Not by benefits alone}

Even though general cognitive flexibility is well correlated with brain size across taxa, this idea is not fully satisfactory as an evolutionary explanation of variation in brain size. For instance, species that rely on extractive foraging may cope better with seasonality, but why did only some of the species living in equally seasonal habitats respond this way? To put it more generally, would not most primates, or indeed animals generally, benefit from being smarter, if there were no countervailing costs of evolving larger brains? We would like to predict which species respond to selective pressures thought to be present quite broadly.

The solution to this conundrum may be that to more fully understand brain size distribution, we need to pay special attention to the costs of changes in brain size. As already mentioned, costs are especially relevant in the case of brains because brain tissue is among the most energetically expensive tissues of the body, ${ }^{3}$ and its energy consumption rate varies only slightly between various brain regions or during different activities. ${ }^{29}$ Thus, the energy costs of the brain cannot be temporarily reduced without risking permanent damage. ${ }^{30}$ The overall energy costs of brains are surprisingly high. Humans spend about $20-25 \%$ of their resting metabolism on the brain (neonates even over 60\%), and costs are also considerable for other animals, especially small ones. ${ }^{31}$ Increased brain size generally leads to cognitive benefits, but whether they also produce a net fitness benefit depends on whether the energetic costs of an increase in brain size are met or they generate a sufficient survival or reproductive benefit to outweigh these energetic costs.

Building on earlier hypotheses ${ }^{4,32,33}$ we proposed the Expensive Brain framework, ${ }^{34}$ which postulates that the costs of a brain that is relatively larger than that of the ancestral species can be met by a stable increase of the overall net energy input, or by changing the allocation of energy to other functions such as production, digestion, or locomotion (lower part of Figure 2). These options are non-exclusive, and their feasibility is largely determined by the taxon-specific characteristics of ecology and lifestyle. Socioecological factors affect a species' potential to follow one or the other of these pathways to pay for an evolutionary 
increase in brain size. Moreover, changes in habitat or lifestyle can occur independently from cognitive traits, but they nevertheless can change the energy budget of a species, or shape the reaction space of the evolutionary feedback loops. We refer to such extrinsic modifications of the various pathways as "boosters" or "filters" (Figure 2). In the following, we review the available comparative evidence for the cost side of explaining brain size variation. Although we will first look at each pathway separately, we also integrate previous hypotheses and findings into the new combined framework, which allows us to better understand detailed interactions of the traits involved.

\section{Increasing net energy intake}

The first pathway to evolve a larger brain is an increase in basal metabolic rate (BMR), ${ }^{4}$ which must be stable over time to avoid brain starvation (Figure 2). This can be achieved by a change of diet towards food items of higher quality, i.e. eating food that has higher energetic value or is easier to digest, ${ }^{32,33,35}$ provided that the concomitant increase in foraging effort is not too large. Net energy intake rate is the difference between the rates of energy intake from food and the energy costs of acquisition per time. Although not every change in dietary intake is necessarily reflected in a change of the basal metabolic rate of a species,${ }^{36}$ we found a significant correlation between BMR and diet quality ${ }^{65}$ in primates if the existance of two hypometabolic clades Loridae and Lemuridae is taken into account ( $\mathrm{N}=29$ primate species, phylogenetic least-squares regression, BMR as response, body mass and hypometabolism as covariates: $\mathrm{F}=402.5$, diet quality $\mathrm{p}<0.001$, estimate $=0.596$; data from ${ }^{38}$ and $\left.{ }^{69}\right)$.

Across placental mammals, we found a significantly positive correlation between BMR and brain size, controlling for phylogenetic relations and body mass. ${ }^{37}$ Using additional measurements of BMR in primates and a new compilation of primate endocranial volumes of more than 7000 skulls, we then found that differences in BMR explain up to $35 \%$ of the variation in relative brain size within primates, or vice versa. ${ }^{38}$ Because it will happen only 
rarely that a change in external environment alone, without any cognitive changes, will improve diet quality, this interspecific correlation between BMR and brain size is a classic case of correlated evolution, in which each variable depends on the other and no causal direction can be identified.

\section{Allocation tradeoffs}

The most renowned allocation tradeoff with regard to brain size, the Expensive Tissue hypothesis, suggests an energetic tradeoff between brain tissue and the size of the digestive tract. It was proposed by Aiello and Wheeler ${ }^{32}$ to explain the enormous degree of encephalization in humans. The original study reported a negative correlation between gut mass and brain size in 18 anthropoid primate species, but the authors themselves recognized that the methodology was not optimal. ${ }^{39}$ Comprehensive comparative studies in bats ${ }^{40}$ and birds $^{41}$ did not find the tradeoff, but energetic costs of digestion would perhaps not be expected to play much of a role in flying animals that are known for very short food retention times. Using a new sample of matching organ mass and endocranial volume data for 100 mammalian species (including 23 primates), we recently rejected the validity of the Expensive Tissue hypothesis as a general principle in mammals or primates. ${ }^{42}$ However, it may still explain the special case of humans as compared to great apes, or part of the brain size variation in other taxa. Indeed, in an artificial laboratory setting, guppies selected for large brains exhibit a reduced size of the gut. ${ }^{43}$ Even so, although selection experiments can yield a proof-of-principle for a functional link between brain size and another trait, their results need not necessarily explain the evolutionary history of species under specific natural conditions, especially if all other evidence points in the opposite direction. The case therefore remains open. Moreover, selection experiments are only feasible for a few small, fastreproducing model species such as fruit flies or guppies, but the adaptive responses in large, long-lived, and socially bonded animals may not be the same as those found in these model species. ${ }^{44}$ Therefore, the alternative approach - a broad comparative approach of comparing 
species or populations using a phylogenetically informed statistical method-remains indispensible.

Although Aiello and coworkers ${ }^{39}$ considered this less likely than a gut-brain tradeoff, we proposed ${ }^{41}$ that a tradeoff with another expensive organ such as the liver, or the summed weight of all visceral organs, or a more abundant, but less expensive tissue such as muscles, could also be used to pay for the increased energy consumption of the brain in relatively large-brained species. In birds, for instance, brain size is negatively correlated with pectoral muscle mass, controlling for phylogenetic relatedness and body mass. ${ }^{41}$ (A re-analysis of this data, this time controlling for body mass minus the mass of the involved organs, confirms the result (PGLS of residuals brain mass vs. residuals pectoral muscle mass, $\mathrm{N}=194$, lambda $=0.930, \mathrm{p}=0.011)$.) Although muscles do not use up much energy during rest, a conflict between the supply of the brain and the flight muscles could exist during peak performance, i.e. during takeoff, or during sustained performance of muscles over a longer period of time, e.g. migration. Further preliminary support of this idea comes from the finding that primates are under-muscled compared with other mammals, ${ }^{45}$ despite the finding that exercise capacity as proxied by maximum metabolic rate is positively correlated with relative brain size in mammals. ${ }^{46}$ Empirical validation of such direct tradeoffs is often hampered by the lack of high-quality data for a sufficient number of species, especially as proper tests would also need to include a measure of energy throughput such as the basal metabolic rate. But as artificial selection experiments demonstrate their existence, we should not neglect their potential role in explaining the evolutionary history of a species, and make an effort to collect more data. Indeed, further insight into a potential gut-brain tradeoff during human evolution critically depends on the availability of detailed body composition data of great apes.

In sum, the pathway of paying for increased brain size by an allocation tradeoff seems most feasible when a rather drastic change in lifestyle (e.g. more digestible diet, cheaper locomotion, etc.) alleviates the negative fitness effects of a reduced allocation to these competing functions, provided that the shift in lifestyle did not rely on improved 
cognitive abilities. This may explain why such adaptive shifts are expressed mostly between higher-level taxa (hence the grade shifts), whereas they do not occur frequently enough within an adaptive radiation of related species to show up in a phylogenetically informed comparative study.

\section{Brain size and production}

Another possible tradeoff exists between allocation to the brain and to production, i.e. growth and reproduction, ${ }^{47}$ which are among the energetically most costly functions within an individual's lifetime. We therefore proposed a tradeoff between brain size and production, both at the level of mothers (as breeding is the most strenuous period for an adult female $\operatorname{animal}^{48}$ ), and at the level of the offspring (because immatures are relatively larger-brained than adults and growing and differentiating brains require even more energy to grow and be maintained ${ }^{49}$ ). Guppy females selected for relatively large brains produced fewer offspring than the smaller-brained lines. ${ }^{43}$ Experiments on fruit flies even demonstrated an induced cost of learning, as learning trials reduced subsequent fertility. ${ }^{50}$ Supporting correlations between brain size and development have also been found in both mammals and birds. ${ }^{51,52}$

To derive a more precise prediction of the Expensive Brain framework, we consider variation in another, partly independent dimension of life history (in addition to the fast-slow continuum), namely developmental state at birth (precocial or altricial). Because altricial species usually produce many young and precocial ones singletons, developmental state is correlated with litter size. ${ }^{53}$ In contrast to other hypotheses, the expensive brain idea predicts that the operation of the brain size-production tradeoff differs between development modes. Indeed, we found that in large-brained precocial mammals the single offspring grows more slowly, whereas large-brained altricial mammals reduce reproductive investment by reducing litter size. ${ }^{34}$ They do not need to prolong development periods, as in most species the constraint is energetic and not temporal. ${ }^{54}$

Enhanced cognition may only be able to produce a fitness benefit if survival is 
sufficiently high, and thus if it is accompanied by offspring quality. The latter can be proxied by the relative size of neonates, as relatively larger size improves their chances of survival. ${ }^{53}$ Indeed, while altricial and precocial mammals do not show an overall difference in relative brain size, within each group larger-brained species produce larger neonates and thus have a prolonged gestation period. ${ }^{34} \mathrm{We}$ even expect that neonate mass is still positively correlated with brain size, if differences in adult mortality are statistically controlled for by including maximum lifespan as a covariate (see below; cf. Figure 3).

\section{The life history filter}

In theory, the reduced reproductive rate of relatively large-brained species could be fully offset by increased survival and a prolonged reproductive lifespan of large-brained species. As discussed above, larger-brained species gain a fitness benefit if their enhanced cognitive flexibility translates into improved survival (upper part of Figure 2). Thus we would not expect a lineage to evolve larger brains if a high level of unavoidable mortality prevents a positive fitness effect of enhanced cognition. ${ }^{17}$ This "life history filter" explains some grade shifts in relative brain size between taxa, such as arboreal and ground-living squirrels, which differ mainly in their predation risk. ${ }^{55}$ It may also explain why in mammals overall, and within primates as a group, relatively larger-brained species are mostly found within the larger-bodied taxa.

For selection to favor increased brain size, the larger-brained individuals must have higher net fitness than the smaller-brained ones in the population. While this must be true, empirically it turns out that the maximum potential reproductive rate (known in ecology as $\left.\mathrm{r}_{\max }\right)^{56}$ is reduced as brain size increases. This is because the reproductive lifespan is not sufficiently prolonged in larger-brained mammals to completely compensate for the reduced annual reproductive rate. ${ }^{57}$ As a consequence, the macroevolutionary trend toward ever increasing brain size noted by Jerison ${ }^{58}$ is counteracted by the constraint that populations of larger-brained species must still be demographically viable. For any given lineage, a 
maximum brain size (a "gray ceiling") therefore exists, beyond which populations reproduce so slowly that they are very susceptible to the risk of going extinct, because they cannot recover from crashes or respond genetically to rapid changes in optimum phenotypes. ${ }^{59}$ Even species with very low extrinsic mortality rates, such as extant orangutans, ${ }^{60}$ are threatened by their limited ability to respond genetically to novel adaptive challenges.

But how can a taxon with a large brain size for its lineage break through the gray ceiling of its lineage? We suspect that the most feasible way is a change in lifestyle toward distributing the costs of offspring production over more individuals than just the mother. Indeed, we found that a booster, energy subsidies during breeding, in the form of allomaternal help, alleviates the tradeoff between production and brain size ${ }^{61,62}$ In placental mammals as a group, and within both terrestrial Carnivora and Rodentia, the amount of allomaternal care is positively correlated with brain size, controlling for several potentially confounding variables such as diet and nocturnality. ${ }^{61}$ Thus, the evolution of cooperative breeding generally favors increased brain size. In nonhuman primates, however, energy subsidies from allomaternal care are invested into an increased reproductive rate, while brains are even smaller than in independently breeding species. ${ }^{61}$ The best explanation for this anomaly is that among nonhuman primates, help mainly comes in the form of infant carrying, and because they are precocial mammals, a large proportion of brain growth occurs in utero, during which help is not effective.

\section{The energy filter}

Since, as noted earlier, the brain depends on a continuous supply of energy and may suffer permanent damage from starvation, ${ }^{30}$ temporal stability of dietary intake is another factor that should affect brain size evolution. In selection experiments, fruit flies selected for high learning ability produced larvae that were less resistant to adverse conditions, ${ }^{63}$ whereas fruit flies selected on their ability to survive environmental stress performed worse in learning tasks. ${ }^{64}$ Thus, we predicted that between species, relative brain size is negatively correlated 
with the degree of seasonality in energy intake, as assessed by variation in the energy content of the diet. This was confirmed for all major primate grades: strepsirrhine primates, ${ }^{65}$ Old World primates, ${ }^{66}$ and New World primates. ${ }^{67}$

In the latter two lineages, we also found a cognitive buffer effect, in that relative large-brained species exhibit less seasonal variation in diet composition than the seasonality of their habitat would suggest. In these analyses, we distinguished between environmental seasonality of the habitat, and the degree of seasonality that is experienced by the animals. While the latter reflects the actual energetic constraint and is thus always negatively correlated with brain size, cognitive buffering can reduce or even cancel the effect of habitat seasonality on brain size. In some birds, the resulting correlation between brain size and habitat seasonality is even positive. ${ }^{20}$ Thus, the most striking implication of our results ${ }^{66}$ is that an energetic constraint can coexist with a cognitive buffer effect. However, the evolution of relatively large brains in seasonal environments, as predicted by the Cognitive Buffer hypothesis, is not found among primates and only expected if the energetic constraint is weak.

Some lemur species are renowned for buffering the extreme fluctuations of their habitat by physiological strategies such as daily torpor or hibernation during the dry season. ${ }^{68}$ To survive, they rely on fat storage. Indeed, across all mammals, we noted a consistent negative correlation between the mass of adipose depots and brain size. ${ }^{42}$ This negative correlation was most strongly expressed in specimens of wild provenance and in females (and thus especially in wild-derived females). We therefore proposed that most mammals follow one of two usually incompatible strategies to avoid starvation in lean periods: either a physiological buffer involving fat storage, or a cognitive buffer involving cognitive flexibility to find food during lean periods. Although fat tissue is not metabolically costly, its cost consists in having to be carried around, which increases locomotion costs and hampers swift escapes from predators. Combining the two strategies would therefore only be possible if the costs of transport of additional body fat are small, for example in aquatic animals or perhaps even bipeds. ${ }^{42}$ As the negative correlation between fat deposits and brain size was found as a 
side result of our test of the Expensive Tissue hypothesis, this result warrants validation from an independent sample.

\section{A combined analysis}

To sum up so far, the strong comparative support for the various predictions of the Expensive Brain framework makes it plausible that brain size could only increase where the animals involved could either achieve a stable rise in overall energy throughput, reduce energy allocation to a competing function, or change their lifestyle to ameliorate the tradeoff between production and brain size. Only then will a cognitive benefit, in the social or ecological domain, be translated into a fitness benefit, and thus only then will selection be able to favor an increase in brain size. Further, when these conditions are satisfied, high social tolerance or prolonged contact between offspring and parents or other tolerant adults would lead to even stronger selection on increased cognitive abilities, and thus brain size. ${ }^{17}$ To test the explanatory power of this framework, we conducted a multivariate, combined analysis of the effects of the various factors that have been shown to correlate with relative brain size across nonhuman primates.

Note that the factors depicted in Figure 2 can no longer be regarded as reflecting either cost or benefits, due to co-evolutionary processes. Maximum lifespan, for example, is on one hand the result of decreased mortality due to the life-saving effect of enhanced cognitive abilities (and thus a benefit of larger brains), but on the other hand it can be used as a proxy for extrinsic mortality risks (and thus a filter preventing the evolution of larger brains in species with high unavoidable mortality). Likewise, higher diet quality can be seen as a benefit if larger brained species manage to obtain better food through cognitive means, or as an energy booster, if the change in diet is brought about by extrinsic changes in food availability that are not affected by cognition. While we do not insist on categorizing each factor according to its role in the framework, it is nevertheless crucial to include several explanatory variables in a test model, even if they are only proxies of the "true" underlying 
factors. Otherwise, the hidden effects of neglected factors are likely to either result in spurious findings or in masking valid correlations. In our view, the best approach to model correlated evolution is a multiple regression with brain size as the response variable (path analysis does not seem justified in this case, as correlated evolution leads to the loss of unidirectional causality, and niche construction to the blurring of external and internal factors).

However, a straightforward model selection approach is difficult because some variables are available only for a subset of species. We therefore applied a stepwise procedure that considers both the explanatory power and the reduction in sample size that arises from the inclusion of an additional predictor variable.

In a first model, only two variables were included: maximum lifespan, reflecting the extrinsic mortality risk toward which the investment in maintenance and repair of an organism is optimized, and basal metabolic rate, which represents the metabolic throughput of an organism at rest. Both these variables reflect the degree to which organisms could pass the life-history and energy filters. However, since BMR is available only for a limited sample of primates and reliable data are especially scarce for large-bodied haplorhines, we also conducted an alternative analysis in which the energy level is proxied by dietary quality and annual stability of diet composition as defined before. ${ }^{65}$ Using diet quality instead of BMR yields a larger sample, which makes it possible to include other variables that may affect variation in brain size, such as neonate mass (as a proxy of infant mortality risk), allomaternal energy subsidies (as a proxy of buffering the energetic bottleneck during the most strenuous reproductive period), or group size (as a proxy of the opportunities for social learning of juveniles ${ }^{17}$ ). Because the various hypotheses postulate the action of various, mutually compatible processes, our framework predicts that a combination of these factors should provide a better explanation for variation in relative brain size than any single factor. In a model selection procedure, models with differing numbers of predictor variables can be compared, as long as the same sample size is equal. Moreover, if human characteristics fit the 
pattern predicted by the primate model, including humans in the model should increase the explained proportion of brain size variation even further.

The first model, including only maximum lifespan and BMR, explains about $46 \%$ of variation in relative brain size ( $\mathrm{N}=28$ species). In the second, larger set of models (Figure 3 ), where BMR was proxied by diet quality, maximum lifespan and diet quality explain about $43 \%$ of the variation in relative brain size of nonhuman primates. Replacing diet quality by annual stability of diet composition yields an $\mathrm{r}^{2}$ of $51.5 \%$ (including both these variables yields a non-significant contribution of diet quality, as they are largely collinear in this sample). With the additional variable neonate mass, the proportion of variance explained increases up to a total of 79\%. In an analogous phylogenetically informed model, both allomaternal care and group size also exert a significant effect on brain size, although their inclusion increases $\mathrm{r}^{2}$ only slightly to $82.5 \%$.

This sequence of models shows that the effects on brain size are additive and nonexclusive, as expected. That the social factors (allomaternal care, group size) explain only a modest amount of variation is also not unexpected, because potential socio-cognitive benefits should be widespread among primates, but in the end it is the ability to afford the energetic costs of larger brains that determines the extent to which lineages can actually realize these benefits.

These findings underscore the importance of ecology in primate brain size evolution: in most cases having larger brains requires that individuals meet the increased energy demands by improving diet or reducing seasonality or they must translate cognitive benefits into survival benefits, often by reducing starvation or predation. Consequently, we suspect that some of the social benefits of larger brains, amply documented among primates, ${ }^{10}$ must have an important ecological component. Indeed, survival and reproduction are known to be improved by the ability to form strong social bonds, ${ }^{70}$ and presumably effective pair bonds in other mammals or birds, ${ }^{11}$ and by social learning of a variety of ecological skills. ${ }^{71}$

When we include humans into the analysis, the proportion of variance explained by 
the model is about 5\% larger in most models. In particular, adding allomaternal care and group size does increase $\mathrm{r}^{2}$ even more, up to a total of $86 \%$ of explained variation in relative brain size (Figure 3). This indicates that our exceptionally large-brained species exhibits a similar suite of traits that is also correlated with large brains in primates in general.

\section{A scenario for human encephalization}

Building on these results, we will now conclude this review by looking into the evolutionary history of hominins to identify processes relevant to hominin evolution that are known to affect the energy available to the brain. We also attempt to find the order in which they appeared and discuss potential correlates of human brain size that have not been included in the comparative model, because they either do not exist in nonhuman primates (efficient bipedalism) or sufficient data are lacking (fat storage).

Regarding the evolution of our own lineage, there is archeological and fossil evidence for various factors that, at one point or another, should have permitted an increase in relative brain size during hominin evolutionary history (Table 1). Although the available evidence is too scarce to allow a detailed matching and timing of the events, we argue for a scenario in which the evolution of traits follows a specific order. In the hominin lineage, brain size has continuously increased, also relative to body size, during the past 2 million years, albeit not in a linear fashion and possibly involving major shifts in the cognitive niche. ${ }^{72}$ Thus, it is easy to imagine positive feedback process involving reduced mortality due to reduced predation, improved diet quality, and reduced experienced seasonality. But it is harder to identify the factor or factors that pushed our ancestors into this co-evolutionary feedback loop. It seems that technology and cooperation were effectively used in the hunting or scavenging context as early as 2.5 million years ago (although with unknown frequency), ${ }^{73}$ while we detail below how prosocial care and efficient locomotion are documented only at 1.8 million years. However, this order may be due to a taphonomic bias, because the time period between 2.5 and 2 million years ago yielded various archeological remains such as stone tools and cut 
marks, whereas fossil remains of Homo are scarce and their affiliations often disputed. ${ }^{74}$ On the other hand, evidence for any of these features in australopithecines is either very indirect (such as large neonates, inferred from adult brain size ${ }^{75}$ ), or their implications are not widely accepted (such as the reduced canine honing complex in Ardipithecus ramidus as evidence for pair bonds ${ }^{76}$.

It seems reasonable to conclude that at around 1.8 million years ago, by which time Homo erectus/ergaster was well established, a distinct human-like combination of traits had developed, which included a more efficient bipedalism, ${ }^{77,78}$ cooperative hunting of big game or confrontational scavenging in large groups, ${ }^{73,79,80}$ and the use of weapons as defense against predators. A rare combination of cognitive buffering (e.g. by extractive foraging and tool use) and physiological buffering by an increased amount of body fat ${ }^{81,82}$ allowed early humans to thrive in the increasingly seasonal habitats of the early Pleistocene Africa, ${ }^{82}$ and keep their new diet composition relatively stable across seasons. ${ }^{83,84}$

It remains to be studied whether efficient bipedalism played a role in making such an unusual combination feasible, or whether reduced energy expenditure for locomotion ${ }^{85}$ was simply achieved by a marked reduction of the energetically demanding climbing. ${ }^{86}$ In our lineage, the total daily energy expenditure increased, ${ }^{87}$ but was there also an increase in basal metabolic rate (BMR) relative to the ancestral state of the common ancestor with Pan? The only available data for nonhuman hominoid BMR stem from immature or young adult chimpanzees (out of 27 individuals, most were infants younger than 3 years, seven individuals labeled as adults were between 6 and 10 years, and only five individuals were between 10 and 15 years $^{88}$ ) that probably had a much lower proportion of body fat than the average human, ${ }^{89}$ which thus yields an overestimate of chimpanzee BMR. A recalculation of Bruhn's original data ${ }^{88}$ yields an average daily BMR of $1250 \mathrm{kcal}$ for a $50 \mathrm{~kg}$ chimpanzee. Taking into account the likely difference in the proportion of body fat, a 39-50kg chimp uses about 1080 to $1250 \mathrm{kcal}$ for basal sustenance per day, whereas a contemporary human of the same fat-free body mass (36-46kg) uses between 1230 and $1500 \mathrm{kcal}$ daily (data of !Kung 
and Ache taken from ${ }^{90}$ ). In conclusion, the only available data on chimpanzee BMR indeed suggest that not only daily energy expenditure, but also BMR relative to fat-free body mass increased at one point during hominin evolution.

Nevertheless, the question remains how the coevolutionary cycle of changes toward the technology-dominated hominin foraging niche and increased brain size got started. To explain convincingly why only the hominin lineage, but not other great apes, leaped into a new technology-based co-evolutionary loop, we must look for a trait that is only found in humans, but whose origin did not depend on the previously evolved presence of enhanced cognitive abilities. Two candidates are available: cooperative breeding and cooperative hunting.

Based on a model of extant primates, we found that intensive allomaternal care, including provisioning of both mothers and dependent offspring, could have allowed ancestral humans to expand their brain beyond the gray ceiling of ape brain size, which we tentatively and conservatively estimated to be about $600-700 \mathrm{cc}^{91}$ Cooperative breeding can evolve in relatively small-brained primates,${ }^{92}$ making it a good candidate for an external booster that allowed us to enter a new co-evolutionary feedback loop.

It might be objected that cooperative breeding and prosocial tendencies are usually restricted to family-living mammals with social monogamy. ${ }^{93}$ Because extant humans are overwhelmingly non-monogamous, ${ }^{94}$ and sexually dimorphic in size, yet show the extensive allomaternal care expected from cooperative breeders, we suspect that cooperative breeding in the hominin lineage showed a different historical trajectory.

The routine acquisition of large amounts of meat, beginning at ca 2.5 millions years $\mathrm{ago}^{73}$ and probably increasing in importance by the time of Homo erectus, ${ }^{79,80}$ implies some level of cooperative hunting or scavenging, which inevitably led to food sharing, simply because the cooperating males could not eat large packages of meat in one sitting. Thus, a change in diet toward high-quality foods that could effectively be shared with others, presumably including female friends (pregnant or lactating) and their offspring, would have 
led to an amelioration of the production tradeoff with brain size This scenario would let extensive allomaternal care arise from cooperative hunting.

Among extant foragers, provisioning and babysitting by post-reproductive females and communal nursing among mothers are additional and important components of allomaternal care. ${ }^{95}$ In primates, as opposed to the situation in other mammals, communal breeding (the sharing of duties among breeding females) exhibits a positive correlation with brain size ${ }^{61}$ Female bonding and eventually grandmothering ${ }^{95}$ are therefore probably also part of the picture of human brain size evolution. At present, we cannot say when grandmothering and the concomitant lengthening of post-reproductive lifespan arose in the human lineage. Although teeth eruption patterns suggest that the modern human-like developmental pace evolved very recently, ${ }^{96}$ this was not necessarily closely linked to the evolution of menopause. Available comparative evidence is of limited use to resolve this issue as communal care by other breeding females and care by males and older siblings are rather independent dimensions of allomaternal care in mammals and primates. ${ }^{61}$ To gain more insights, future comparative studies will need to carefully define and evaluate the dimensions of cooperative and communal breeding, and cooperative hunting in the few animal lineages that exhibit a combination of these traits.

In conclusion, the archeological evidence for big-game hunting or scavenging at an early stage in the evolution of the genus Homo points towards a major role of cooperative hunting, which led to male food sharing, as a trigger of the remarkable increase in hominin brain size. Provisioning and babysitting by post-reproductive females and communal nursing among breeding females may have pre- or postdated this change in lifestyle, but from the comparative evidence ${ }^{61}$ we suspect that female help on its own would not have allowed for the evolution of the uniquely human combination of traits that arose between 2.5 and 2 million years ago. 


\section{Acknowledgments}

We thank all the people who generously contributed data to our compilations, and Ana Navarrete and Janneke van Woerden for their major contribution to data collection. Financial support was provided through Swiss National Science Foundation grants No. 3100A0-117789 and 31003A-144210) and the A.H. Schultz foundation.

\section{$\underline{\text { References }}$}

1 Deaner RO, Isler K, Burkart JM, van Schaik CP. 2007. Overall brain size, and not encephalization quotient, best predicts cognitive ability across non-human primates. Brain Behav Evol 70:115-124.

2 Reader SM, Hager Y, Laland KN. 2011. The evolution of primate general and cultural intelligence. Phil Trans Roy Soc B Biol Sci 366:1017-1027.

3 Niven JE, Laughlin SB. 2008. Energy limitation as a selective pressure on the evolution of sensory systems. J Exp Biol 211:1792-1804.

4 Martin RD. 1981. Relative brain size and basal metabolic rate in terrestrial vertebrates. Nature 293:57-60.

5 Byrne RW, Whiten A. 1988. Machiavellian Intelligence: Social Expertise and the Evolution of Intellect in Monkeys, Apes, and Humans. Oxford, Clarendon Press.

6 Dunbar RIM, Shultz S. 2007. Understanding primate brain evolution. Phil Trans Roy Soc B Biol Sci 362:649-658.

7 Odling-Smee FJ, Laland KN, Feldman MW. 1996. Niche construction. Am Nat 147:641648.

8 Shultz S, Dunbar R. 2010. Encephalization is not a universal macroevolutionary phenomenon in mammals but is associated with sociality. Proc Natl Acad Sci USA 107:21582-21586. 
9 Deaner RO, Barton RA, van Schaik CP. 2003. Primate brains and life histories: Renewing the connection. In: Kappeler PM, Pereira ME, editors. Primate Life Histories and Socioecology. Chicago, Chicago University Press. p 233-265.

10 Shultz S, Dunbar RIM. 2007. The evolution of the social brain: Anthropoid primates contrast with other vertebrates. Proc Roy Soc B Biol Sci 274:2429-2436.

11 Shultz S, Dunbar RIM. 2010. Social bonds in birds are associated with brain size and contingent on the correlated evolution of life-history and increased parental investment. Biol J Linn Soc 100:111-123.

12 Byrne RW. 1997. The technical intelligence hypothesis: an additional evolutionary stimulus to intelligence. In: Whiten A, Byrne R, editors. Machiavellian Intelligence II, Cambridge University Press. p 289-311.

13 Gibson KR. 1986. Cognition, brain size and the extraction of embedded food resources. In: Else JG, Lee PC, editors. Primate Ontogeny, Cognition, and Social Behaviour. Cambridge, Cambridge University Press. p 93-105.

14 Zuberbühler K, Byrne RW. 2006. Social cognition. Curr Biol 16:R786-R790.

15 Dunbar RIM. 1998. The social brain hypothesis. Evol Anthropol 6:178-190.

16 Holekamp KE. 2007. Questioning the social intelligence hypothesis. Trends Cogn Sci 11:65-69.

17 van Schaik CP, Isler K, Burkart JM. 2012. Explaining brain size variation: From social to cultural brain. Trends Cogn Sci 16:277-284.

18 Allman J, McLaughlin T, Hakeem A. 1993. Brain weight and life span in primate species. Proc Natl Acad Sci USA 90:118-122.

19 Sol D, Bacher S, Reader SM, Lefebvre L. 2008. Brain size predicts the success of mammal species introduced into novel environments. Am Nat 172:S63-S71.

20 Lefebvre L, Reader SM, Sol D. 2004. Brains, innovations and evolution in birds and primates. Brain Behav Evol 63:233-246.

21 Stearns SC. 1992. The Evolution of Life Histories. Oxford, Oxford University Press. 
22 Martin RD. 1996. Scaling of the mammalian brain: The maternal energy hypothesis. News Physiol Sci 11:149-156.

23 Barrickman NL, Bastian ML, Isler K, van Schaik CP. 2008. Life history costs and benefits of encephalization: a comparative test using data from long-term studies of primates in the wild. J Hum Evol 54:568-590.

24 Barton RA. 2012. Embodied cognitive evolution and the cerebellum. Phil Trans Roy Soc B Biol Sci 367:2097-2107.

25 Iwaniuk AN, Lefebvre L, Wylie DR. 2009. The comparative approach and brainbehaviour relationships: a tool for understanding tool use. Can J Exp Psychol 63:150-159.

26 Swanson EM, Holekamp KE, Lundrigan BL, Arsznov BM, Sakai ST. 2012. Multiple determinants of whole and regional brain volume among terrestrial carnivorans. PloS One $7: e 38447$.

27 Reader SM, Laland KN. 2002. Social intelligence, innovation, and enhanced brain size in primates. Proc Natl Acad Sci USA 99:4436-4441.

28 van Schaik CP, Burkart JM. 2011. Social learning and evolution: The cultural intelligence hypothesis. Phil Trans Roy Soc B Biol Sci 366:1008-1016.

29 Raichle ME. 2006. The brain's dark energy. Science 314:1249-1251.

30 Lukas WD, Campbell BC. 2000. Evolutionary and ecological aspects of early brain malnutrition in humans. Human Nature 11:1-26.

31 Mink JW, Blumenschine RJ, Adams DB. 1981. Ratio of central nervous system to body metabolism in vertebrates - its constancy and functional basis. Am J Physiol 241:R203$\mathrm{R} 212$.

32 Aiello LC, Wheeler P. 1995. The expensive-tissue hypothesis - the brain and the digestive-system in human and primate evolution. Curr Anthropol 36:199-221.

33 Leonard WR, Robertson ML. 1997. Comparative primate energetics and hominid evolution. Am J Phys Anthropol 102:265-281.

34 Isler K, van Schaik CP. 2009. The Expensive Brain: A framework for explaining evolutionary changes in brain size. J Hum Evol 57:392-400. 
35 Carmody RN, Wrangham RW. 2009. The energetic significance of cooking. J Hum Evol $57: 379-391$.

36 Pontzer H, Kamilar JM. 2009. Great ranging associated with greater reproductive investment in mammals. Proc Natl Acad Sci USA 106:192-196.

37 Isler K, van Schaik CP. 2006. Metabolic costs of brain size evolution. Biol Lett 2:557-560.

38 Isler K, Kirk EC, Miller JMA, Albrecht GA, Gelvin BR, Martin RD. 2008. Endocranial volumes of primate species: Scaling analyses using a comprehensive and reliable data set. J Hum Evol 55:967-978.

39 Aiello LC, Bates N, Joffe T. 2001. In defense of the expensive tissue hypothesis. In: Falk D, Gibson KR, editors. Evolutionary Anatomy of the Primate Cerebral Cortex. Cambridge, Cambridge University Press. p 57-78.

40 Jones KE, MacLarnon AM. 2004. Affording larger brains: Testing hypotheses of mammalian brain evolution on bats. Am Nat 164:E20-E31.

41 Isler K, van Schaik CP. 2006. Costs of encephalisation: The energy trade-off hypothesis tested on birds. J Hum Evol 51:228-243.

42 Navarrete AF, van Schaik CP, Isler K. 2011. Energetics and the evolution of human brain size. Nature 480:91-93.

43 Kotrschal A, Rogell B, Bundsen A, Svensson B, Zajitschek S, Brännström I, Immler S, Maklakov AA, Kolm N. 2013. Artificial selection on relative brain size in the guppy (Poecilia reticulata) reveals costs and benefits of evolving a larger brain. Curr Biol 23:168-171.

44 Isler K. 2013. Brain size evolution: how fish pay for being smart. Curr Biol 23:R63-R65.

45 Muchlinski MN, Snodgrass JJ, Terranova CJ. 2012. Muscle mass scaling in primates: An energetic and ecological perspective. Am J Primatol 74:395-407.

46 Raichlen DA, Gordon AD. 2011. Relationship between exercise capacity and brain size in mammals. PLoS One 6:e20601.

47 Charnov EL, Berrigan D. 1993. Why do female primates have such long lifespans and so few babies? or Life in the slow lane. Evol Anthropol 1:191-194. 
48 Speakman JR. 2008. The physiological costs of reproduction in small mammals. Phil Trans Roy Soc B Biol Sci 363:375-398.

49 Kennedy C, Sokoloff L. 1957. An adaptation of the Nitrous Oxide method to the study of cerebral circulation in children - Normal values for cerebral blood flow and cerebral metabolic rate in childhood. J Clin Investig 36:1130-1137.

50 Mery F, Kawecki TJ. 2005. A cost of long-term memory in Drosophila. Science 308:2005.

51 Barton RA, Capellini I. 2011. Maternal investment, life histories, and the costs of brain growth in mammals. Proc Natl Acad Sci USA 108:6169-6174.

52 Iwaniuk AN, Nelson JE. 2003. Developmental differences are correlated with relative brain size in birds: A comparative analysis. Can J Zool 81:1913-1928.

53 Sibly RM, Brown JH. 2009. Mammal reproductive strategies driven by offspring mortality-size relationships. Am Nat 173:E185-E199.

54 Schuppli C, Isler K, van Schaik CP. 2012. How to explain the unusually late age at skill competence among humans? J Hum Evol 63:843-850.

55 Shattuck MR, Williams Sa. 2010. Arboreality has allowed for the evolution of increased longevity in mammals. Proc Natl Acad Sci USA 107:4635-4639.

56 Cole L. 1954. The population consequences of life-history phenomena. Q Rev Biol 29:103-137.

57 Isler K, van Schaik CP. 2009. Why are there so few smart mammals (but so many smart birds)? Biol Lett 5:125-129.

58 Jerison HJ. 1973. Evolution of the Brain and Intelligence. New York, Academic Press.

59 van Schaik CP. 2013. The costs and benefits of flexibility as an expression of behavioural plasticity: a primate perspective. Phil Trans Roy Soc B Biol Sci 368:20120339.

60 Wich SA, Utami-Atmoko SS, Setia TM, Rijksen HD, Schurmann C, van Schaik C. 2004. Life history of wild Sumatran orangutans (Pongo abelii). J Hum Evol 47:385-398.

61 Isler K, van Schaik CP. 2012. Allomaternal care, life history and brain size evolution in mammals. J Hum Evol 63:52-63. 
62 Isler K. 2011. Energetic trade-offs between brain size and offspring production: Marsupials confirm a general mammalian pattern. BioEssays 33:173-179.

63 Mery F, Kawecki TJ. 2003. A fitness cost of learning ability in Drosophila melanogaster. Proc Roy Soc B Biol Sci 270:2465-2469.

64 Kolss M, Kawecki TJ. 2008. Reduced learning ability as a consequence of evolutionary adaptation to nutritional stress in Drosophila melanogaster. Ecol Entomol 33:583-588.

65 van Woerden JT, van Schaik CP, Isler K. 2010. Effects of seasonality on brain size evolution: evidence from strepsirrhine primates. Am Nat 176:758-767.

66 van Woerden JT, Willems EP, van Schaik CP, Isler K. 2012. Large brains buffer energetic effects of seasonal habitats in catarrhine primates. Evolution 66:191-199.

67 van Woerden JT. 2011. The Influence of Seasonality on Brain Size Evoution in Primates [PhD thesis]. Zurich: University of Zurich.

68 Schülke O, Ostner J. 2007. Physiological ecology of cheirogaleid primates: variation in hibernation and torpor. Acta Ethologica 10:13-21.

69 Rowe N, Myers M. 2012. All the World's Primates. Charlestown, RI: Primate Conservation Inc. http://www.alltheworldsprimates.org, accessed on december $17^{\text {th }}, 2012$.

70 Silk JB, Beehner JC, Bergman TJ, Crockford C, Engh AL, Moscovice LR, Wittig RM, Seyfarth RM, Cheney DL. 2010. Strong and consistent social bonds enhance the longevity of female baboons. Curr Biol 20:1359-1361.

71 Galef BG, Giraldeau L-A. 2001. Social influences on foraging in vertebrates: causal mechanisms and adaptive functions. Anim Behav 61:3-15.

72 Whiten A, Erdal D. 2012. The human socio-cognitive niche and its evolutionary origins. Phil Trans Roy Soc B Biol Sci 367:2119-2129.

73 Dominguez-Rodrigo M, Pickering TR, Bunn HT. 2010. Configurational approach to identifying the earliest hominin butchers. Proc Natl Acad Sci USA 107:20929-20934.

74 Anton SC. 2012. Early Homo: who, when, and where. Curr Anthropol 53:S278-S298.

75 DeSilva JM. 2011. A shift toward birthing relatively large infants early in human evolution. Proc Natl Acad Sci USA 108:1022-1027. 
76 Lovejoy CO. 2009. Reexamining human origins in light of Ardipithecus ramidus. Science $326: 74 \mathrm{e} 71-74 \mathrm{e} 78$.

77 DeSilva JM. 2009. Functional morphology of the ankle and the likelihood of climbing in early hominins. Proc Natl Acad Sci USA 106:6567-6572.

78 Pontzer H, Rolian C, Rightmire GP, Jashashvili T, de Leon MSP, Lordkipanidze D, Zollikofer CPE. 2010. Locomotor anatomy and biomechanics of the Dmanisi hominins. J Hum Evol 58:492-504.

79 Sahnouni M, Rosell J, van der Made J, Maria Verges J, Olle A, Kandi N, Harichane Z, Derradji A, Medig M. 2013. The first evidence of cut marks and usewear traces from the Plio-Pleistocene locality of El-Kherba (Ain Hanech), Algeria: implications for early hominin subsistence activities circa 1.8 Ma. J Hum Evol 64:137-150.

80 Balter V, Braga J, Telouk P, Thackeray JF. 2012. Evidence for dietary change but not landscape use in South African early hominins. Nature 489:558-560.

81 Wells JCK. 2012. The capital economy in hominin evolution: How adipose tissue and social relationships confer phenotypic flexibility and resilience in stochastic environments. Curr Anthropol 53:S466-S478.

82 Potts R. 2012. Environmental and behavioral evidence pertaining to the evolution of early Homo. Curr Anthropol 53:S299-S317.

83 Hill KR, Hawkes K, Hurtado MJ, Kaplan H. 1984. Seasonal variance in the diet of Ache hunter-gatherers in Eastern Paraguay. Human Ecology 12:101-135.

84 Hurtado AM, Hill KR. 1990. Seasonality in a foraging society: variation in diet, work effort, fertility, and sexual division of labor among the Hiwi of Venezuela. J Anthropol Res 46:293-346.

85 Pontzer H. 2012. Ecological energetics in early Homo. Curr Anthropol 53:S346-S358.

86 Hanna JB, Schmitt D, Griffin TM. 2008. The energetic cost of climbing in primates. Science 320:898.

87 Pontzer H, Raichlen DA, Wood BM, Mabulla AZP, Racette SB, Marlowe FW. 2012. Hunter-gatherer energetics and human obesity. PloS One 7: e40503. 
88 Bruhn JM, Benedict FG. 1936. The respiratory metabolism of the chimpanzee. Proc Am Acad Arts Sci 71:259-326.

89 Wells JCK. 2010. The Evolutionary Biology of Human Body Fatness. Cambridge UK, Cambridge University Press.

90 Froehle AW, Schoeninger MJ. 2006. Intraspecies variation in BMR does not affect estimates of early hominin total daily energy expenditure. Am J Phys Anthropol 131:552559.

91 Isler K, van Schaik CP. 2012. How our ancestors broke through the gray ceiling: comparative evidence for cooperative breeding in early Homo. Curr Anthropol 53:S453S465.

92 Burkart JM, Hrdy SB, van Schaik CP. 2009. Cooperative breeding and human cognitive evolution. Evol Anthropol 18:175-186.

93 Clutton-Brock TH. 2006. Cooperative breeding in mammals. In: Kappeler P, van Schaik CP, editors. Cooperation in Primates and Humans - Mechanisms and Evolution. Berlin, Springer. p 173-190.

94 Marlowe FW. 2003. The mating system of foragers in the standard cross-cultural sample. Cross-Cultural Research 37:282-306.

95 Hawkes K, O'Connell JF, Blurton Jones NG, Alvarez H, Charnov EL. 1998. Grandmothering, menopause, and the evolution of human life histories. Proc Natl Acad Sci USA 95:1336-1339.

96 Schwartz GT. 2012. Growth, development, and life history throughout the evolution of Homo. Curr Anthropol 53:S395-S408.

97 Semaw S, Renne P, Harris JWK, Feibel CS, Bernor RL, Fesseha N, Mowbray K. 1997. 2.5-million-year-old stone tools from Gona, Ethiopia. Nature 385:333-336.

98 Lepre CJ, Roche H, Kent DV, Harmand S, Quinn RL, Brugal J-P, Texier P-J, Lenoble A, Feibel CS. 2011. An earlier origin for the Acheulian. Nature 477:82-85.

99 Smith BH, Tompkins RL. 1995. Toward a life-history of the Hominidae. Ann Rev Anthropol 24:257-279. 
100 Haeusler M, Schiess R, Boeni T. 2013. Evidence for juvenile disc herniation in a Homo erectus boy skeleton. Spine 38:E123-E128.

101 Gabunia L, Vekua A, Lordkipanidze D, Swisher CC, Ferring R, Justus A, Nioradze M, Tvalchrelidze M, Anton SC, Bosinski G and others. 2000. Earliest Pleistocene hominid cranial remains from Dmanisi, Republic of Georgia: Taxonomy, geological setting, and age. Science 288:1019-1025.

102 Brain CK, Sillen A. 1988. Evidence from the Swartkrans cave for the earliest use of fire. Nature 336:464-466.

103 Berna F, Goldberg P, Horwitz LK, Brink J, Holt S, Bamford M, Chazan M. 2012.

Microstratigraphic evidence of in situ fire in the Acheulean strata of Wonderwerk Cave, Northern Cape province, South Africa. Proc Natl Acad Sci USA 109:E1215-E1220.

104 van Schaik CP, Isler K. 2012. Life history evolution in primates. In: Call J, Kappeler PM, Mitani J, Palombit RA, Silk J, editors. The Evolution of Primate Societies. Chicago IL, University of Chicago Press. p 220-244. 
Table 1: Evidence for traits that allowed for human brain expansion

\begin{tabular}{|c|c|c|c|}
\hline Trait & Energetic effect & Evidence & Since when? \\
\hline $\begin{array}{l}\text { Cooperative } \\
\text { hunting/scavenging }\end{array}$ & $\begin{array}{l}\text { Improved diet quality, } \\
\text { increased stability in dietary } \\
\text { intake, reduced mortality }\end{array}$ & $\begin{array}{l}\text { Human-made cut } \\
\text { marks on large } \\
\text { animal bones }\end{array}$ & $\begin{array}{l}\text { 2.5Mya: Gona }{ }^{73} \text { (North } \\
\text { Africa } 1.78 \mathrm{Mya}^{79} \\
\text { South Africa } 1.9-1.8 \\
\text { Mya }^{80} \text { ) }\end{array}$ \\
\hline \multirow[t]{2}{*}{ Technology } & $\begin{array}{l}\text { Improved diet quality, } \\
\text { increased stability in dietary } \\
\text { intake }\end{array}$ & Stone tools & $\begin{array}{l}\text { 2.6-2.5 Mya Oldowan } \\
\text { 1.76 } \\
\text { Mya Acheulian }\end{array}$ \\
\hline & Reduced mortality in conflicts & $\begin{array}{l}\text { Cooperative use of } \\
\text { weapons }\end{array}$ & no direct evidence \\
\hline \multirow[t]{2}{*}{ Allomaternal care } & $\begin{array}{l}\text { Energy subsidies for mothers } \\
\text { and weaned offspring }\end{array}$ & Large neonates & $\begin{array}{l}\text { Debated: } \\
\text { australopithecines }{ }^{75} \text { or } \\
\text { Homo }^{99}\end{array}$ \\
\hline & $\begin{array}{l}\text { Reduced mortality through } \\
\text { mutual support and defense }\end{array}$ & $\begin{array}{l}\text { Healed debilitating } \\
\text { injuries or chronic } \\
\text { illness }\end{array}$ & $\begin{array}{l}\text { 1.8 Mya: Homo } \\
\text { erectus }\end{array}$ \\
\hline Efficient bipedalim & $\begin{array}{l}\text { Reduced daily energy } \\
\text { expenditure }\end{array}$ & $\begin{array}{l}\text { Modern human-like } \\
\text { postcranial } \\
\text { morphology }\end{array}$ & $\begin{array}{l}\text { 1.8 Mya: Homo } \\
\text { erectus }^{77,78}\end{array}$ \\
\hline Fat storage & $\begin{array}{l}\text { Physiological buffering of } \\
\text { lean periods }\end{array}$ & $\begin{array}{l}\text { Indirect evidence: } \\
\text { climate fluctuations }\end{array}$ & 3-1.5 $\mathrm{Mya}^{82}$ \\
\hline Cooking & Better digestibility of food & Use of fire & $\begin{array}{l}1 \text { Mya: Swartkrans } \\
\text { Wonderwerk cave }^{103}\end{array}$ \\
\hline
\end{tabular}




\section{Figure legends}

Figure 1: A simple model of energetic costs and fitness benefits effects of an evolutionary increase in brain size. While energetic costs may decrease fitness, the benefits of enhanced cognitive performance increase fitness. Both must be considered to assess the net effect of an increase in brain size on fitness.

Figure 2: Benefits, costs and fitness effects of a change toward relatively larger brain size. This graph depicts the pathways through which energetic costs may decrease fitness (red arrows) and through which the benefits of enhanced cognitive performance may increase fitness (yellow arrows). It also shows the selective feedback loops that lead to coevolution of life history, metabolic rate and brain size (blue arrows). The currently observed combinations of traits represent equilibrium points; therefore the arrows do not indicate cause and effect. The balance of costs and benefits is affected by independent processes that reduce benefits (filters) or reduce energy costs (boosters). Changes in lifestyle or habitat may affect the feasibility of following some of these pathways by imposing a filter on their evolution. First, severe climatic variability or digestive adaptations to a high-fiber diet may prevent a stable increase in energy throughput (energy filter). Second, small body size may lead to high extrinsic mortality through predation that cannot be altered by cognitive performance (life-history filter). These filters prevent the translation of cognitive benefits of large brains into fitness benefits, and therefore actually prevent large brains from evolving in the species or lineages affected by these factors. On the other hand, cost reductions should also affect the feasibility of some pathways. Thus, energy subsidies during breeding (allomaternal care), a change in diet towards high-caloric food, or morphological adaptations towards more efficient locomotor style ("boosters" of energy) can reduce or eliminate the negative effect on fitness due to the energetic costs of relatively large brains, and thus facilitate 
the evolution of relatively larger brains in lineages or species that exhibit such a change in lifestyle.

Figure 3: Additive correlates of relative brain size in six multivariate regression models. The graph depicts $r^{2}$-values from multiple regressions of residuals, in which both the response variable brain size, and all effects were controlled for body mass by regressing them against female body mass and using the residuals for further analysis. Humans were not included in these regressions, because they would otherwise cause undue leverage. In analogous phylogenetic models (PGLS, excluding humans, female body mass as covariate, no use of residuals), these same variables were also found to exert significant effects on brain size. Delta AICc denotes the difference in the Akaike's Information Criterion (corrected for small sample size) between a model $n$ and its neighbor to the left (model $n-1$ ), with sample size $\mathrm{N}$ and the phylogenetic structure parameter lambda set to the values estimated for model $n$. A better-fitting model is characterized by its AICc being lower by at least two. Data are taken from ${ }^{38,61,65,66,69,104}$ for primates, and from ${ }^{23,83,84}$ for humans. 
$\underline{\text { Figures }}$

\section{Correlates of larger brain size}

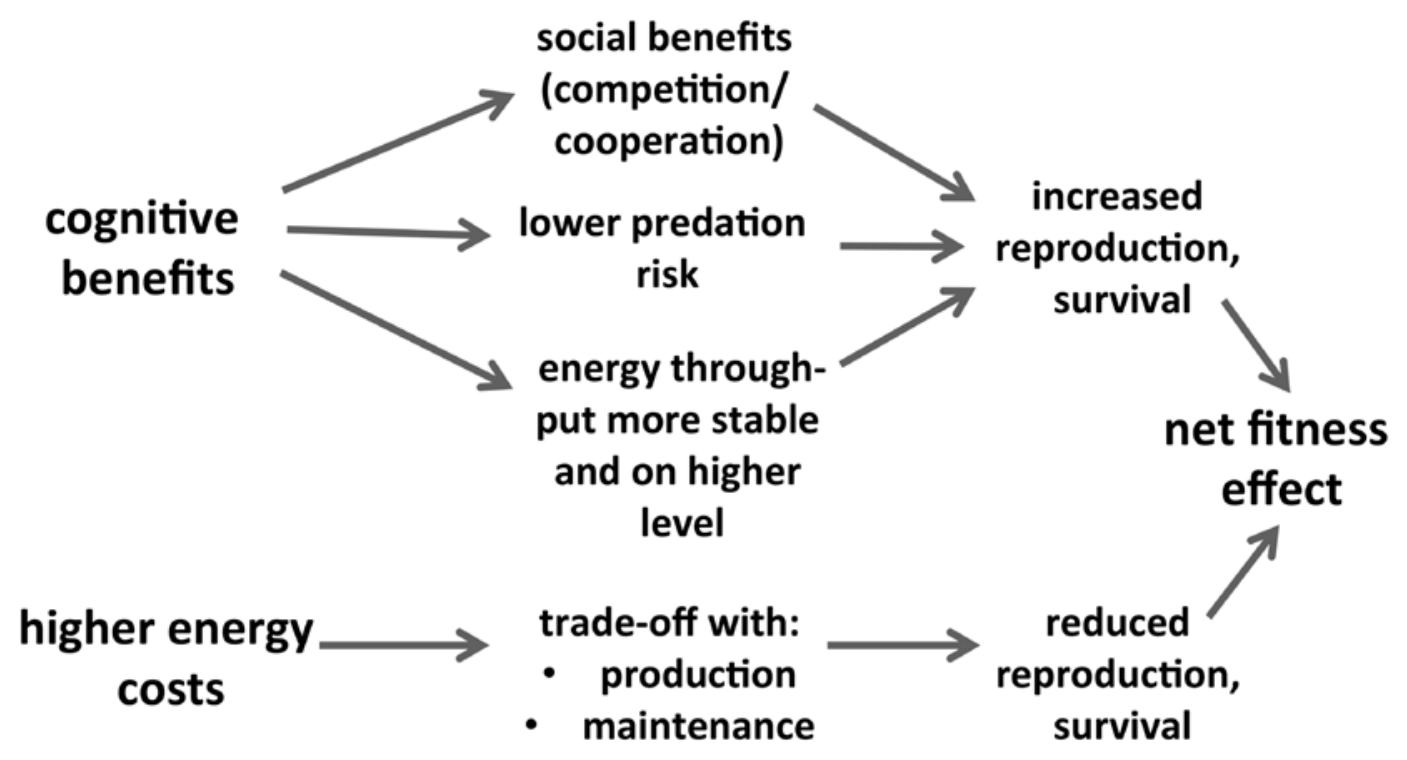

Figure 1 


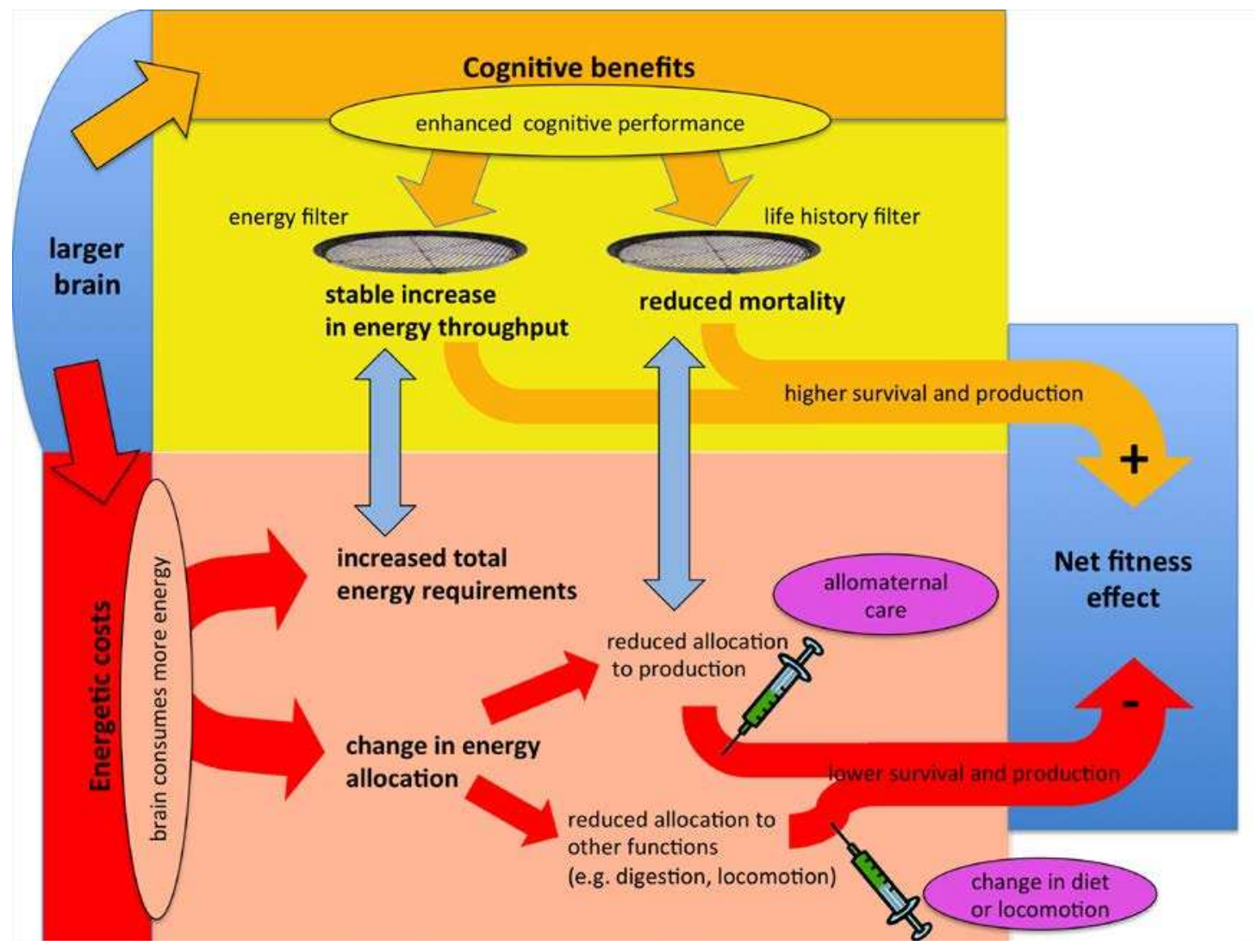

Figure 2 


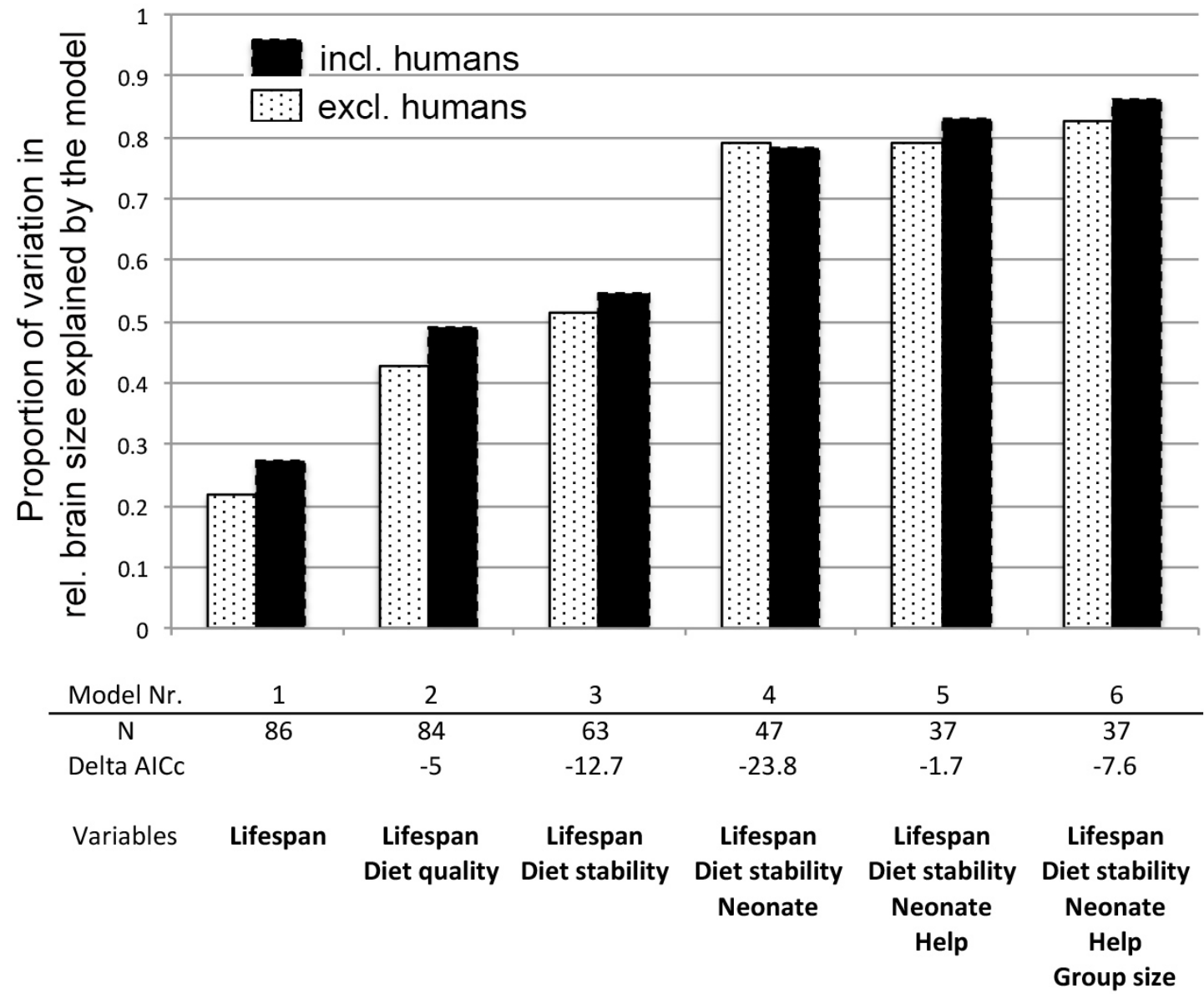

Figure 3 